\title{
General restrictions on tail probabilities
}

\author{
A. De Schepper*, B. Heijnen \\ Faculteit TEW, University of Antwerp (RUCA), Middelheimlaan 1, B-2020 Antwerpen, Belgium
}

Received 12 July 1993; revised 8 May 1994

\begin{abstract}
When limited information on the distribution of a positive random variable $X$ (continuous or discrete) is known (e.g., mode, mean, variance), the tail probability $P(X \geqslant t)$ cannot be chosen independently. In this paper supremum and infimum for $P(X \geqslant t)$ will be calculated over the set of positive random variables with unique mode, mean and/or variance given.
\end{abstract}

Keywords: Tail probability; Incomplete information; Mode; Moments

\section{Introduction}

Because of the developments in the field of computer technology (i.e., reduction of cost, increase of speed, etc.) analytical work has lost a great deal of its interest. However, ignoring some general patterns can be very dangerous and can lead to problems unexplainable by computer logic.

A characteristic example of this phenomenon can be found in [4], where numerical best bounds are derived on a Riemann-Stieltjes integral with respect to a certain distribution function. The underlying random variable $X$ is not known, and only the unique mode, mean, variance, etc. are available. Goovaerts et al. [4] point out, without any explication, that the method fails in case the mode $(=m)$ equals 1 , the mean $\left(=\mu_{1}\right)$ equals 10 and the tail probability $P(X \geqslant 100)$ equals $5 \%$. In the tables with numerical illustrations only an "overflow" message is mentioned.

The reason for this failure is rather fundamental. In fact, the tail probability of $5 \%$ simply is not compatible with $m=1$ and $\mu_{1}=10$ for a positive random variable. One can prove that, in this situation, $P(X \geqslant 100)<4.77 \%$ !

On the one hand, everybody knows that moments of an unknown distribution function cannot be chosen completely freely. Of course, e.g., $E\left(X^{2}\right)$ may not be smaller than $E(X)^{2}$. On the other hand,

\footnotetext{
* Corresponding author.

0377-0427/95/\$09.50 (C) 1995 Elsevier Science B.V. All rights reserved SSDI $0377-0427(95) 00014-3$
} 
however, also mode, moments in combination with tail probabilities cannot be chosen arbitrarily, and this is essentially what happens in [4].

In this paper, the interval which must contain a certain probability $P(X \geqslant t)$ - where $t$ is a given constant in the range of $X$-is determined, if the unique mode $m$, the first and second moments $\mu_{1}$ and $\mu_{2}$ and their range $[0, b]$ are known. In other words, we are looking for the best analytical upper and lower bound for the probability $P(X \geqslant t)$, given $m, \mu_{1}, \mu_{2}$ and $[0, b]$. The results remain valid for $b$ going to infinity.

In the following paragraph, we briefly discuss the method used to derive the extreme values for $P(X \geqslant t)$. Afterwards we give the results in the different cases of known mode and moments. For the detailed derivation of all results, we would like to refer to [2].

\section{Method}

\subsection{Formulation of the problem}

If one wants to determine the boundary values that restrict all possible outcomes for tail probabilities $P(X \geqslant t)=E\left[1_{[t,+\infty[}(X)\right]$, the problem in fact is to find

$$
\sup _{F \in \Phi} \int_{0}^{b} 1_{[t,+\infty[}(x) \mathrm{d} F(x) \text { and } \inf _{F \in \Phi} \int_{0}^{b} 1_{[t,+\infty[}(x) \mathrm{d} F(x),
$$

where $\Phi$ is the class of all distribution functions with range $[0, b]$ and with mode $m$ and moments $\mu_{1}$ and $\mu_{2}$ if known.

\subsection{Use of the knowledge of the mode}

Lemma 1. If a unimodal random variable $X$ has range $[0, b]$, mode $m$ and moments $\mu_{1}$ and $\mu_{2}$, then there exists a random variable $Y$ with same range $[0, b]$ and with moments $v_{1}=2 \mu_{1}-m$ and $v_{2}=3 \mu_{2}-2 m \mu_{1}$, such that for any function $g(x)$ the following equality holds:

$$
E[g(X)]=E[f(Y)],
$$

where

$$
f(x)=\frac{1}{x-m} \int_{0}^{x-m} g(\xi+m) \mathrm{d} \xi \quad \text { ("Khinchine transform"). }
$$

Proof. The proof of this result can be found in Khinchine's famous characterization of unimodality [3].

In the case of the tail probabilities, one has $g(x)=1_{[t, b]}(x)$, which implies a Khinchine transform

$$
f(x)= \begin{cases}\frac{x-t}{x-m} 1_{[t, b]}(x) & t>m, \\ \frac{t-m}{x-m} 1_{[0,[}(x)+1_{[t, b]}(x) & t \leqslant m\end{cases}
$$


and as a consequence the problem now is to find

$$
\sup _{F \in \Psi} \int_{0}^{b} f(x) \mathrm{d} F(x) \text { and } \inf _{F \in \Psi} \int_{0}^{b} f(x) \mathrm{d} F(x),
$$

where $\Psi$ is the class of all distribution functions with range $[0, b]$ and moments $v_{1}$ and $v_{2}$ if known.

\subsection{Basic reasoning}

If $F \in \Psi$ and if $P(x)$ is a polynomial of degree 1 (resp. 2) or less, then the value of $\int_{0}^{b} P(x) \mathrm{d} F(x)$ only depends on the first moment of $F$ (resp. the first and second moments of $F$ ), and so it is the same for each $F$ and $\Psi$ in case these moments are known.

We now look for such polynomials $P(x)$ greater (resp. smaller) or equal to $f(x)$ on $[0, b]$ and for some distribution $G$ of $\Psi$ for which

$$
\int_{0}^{b} P(x) \mathrm{d} G(x)=\int_{0}^{b} f(x) \mathrm{d} F(x) .
$$

As distribution $G$ we will use one, two or three point distributions of $\Psi$, as polynomial $P(x)$ we will use that polynomial that maches $f(x)$ in the mass-points of $G$, such that Eq. (5) holds.

\subsection{Generation of few points distributions}

Suppose that $X$ is a random variable with range $[0, b]$ and moments $v_{1}$ and $v_{2}$. Define $r^{\prime}=\left(v_{2}-v_{1} r\right) /\left(v_{1}-r\right)$ for $r \in[0, b], r \neq v_{1}$, then $0<b^{\prime}<v_{1}<0^{\prime}<b$, as suggested in [5].

\section{Two point distribution}

If $r \in\left[0, b^{\prime}\right]$, then there exists a unique two point distribution in $\left\{r, r^{\prime}\right\}$ with masses

$$
q_{r}=\frac{v_{1}-r^{\prime}}{r-r^{\prime}} \quad \text { and } \quad q_{r^{\prime}}=\frac{v_{1}-r}{r^{\prime}-r}
$$

Three point distribution

If $r \in\left[b^{\prime}, 0^{\prime}\right]$, then there exists a unique three point distribution in $\{0, r, b\}$ with masses

$$
q_{r}=\frac{b v_{1}-v_{2}}{r(b-r)}, \quad q_{b}=\frac{v_{2}-v_{1} r}{b(b-r)} \quad \text { and } \quad q_{0}=1-q_{r}-q_{b}
$$

\subsection{Essential conditions}

To guarantee the existence of a distribution function on $[0, b]$ with given $b, m, \mu_{1}$ and $\mu_{2}$, this "known" parameters cannot be chosen completely arbitrarily. We have to take into consideration 
the following essential conditions, which can be deduced from elementary conditions on distribution functions:

$$
\begin{aligned}
& b \mu_{1} \geqslant \mu_{2}, \quad \mu_{1}^{2} \leqslant \mu_{2}, \quad 2 \mu_{1} \geqslant m, \\
& 4 \mu_{1}^{2}-2 \mu_{1} m+m^{2} \leqslant 3 \mu_{2}, \quad 2 b \mu_{1}+2 \mu_{1} m-m b \geqslant 3 \mu_{2} .
\end{aligned}
$$

2.6. Calculation of the extreme values

After having determined the few point distribution for which Eq. (5) holds, we can deduce (after (sometimes) tedious calculations) the supremum or infimum as follows:

- one point distribution in $\{r\}$ :

$$
f(r) \cdot q_{r} ;
$$

- two point distribution in $\left\{r, r^{\prime}\right\}$ :

$$
f(r) \cdot q_{r}+f\left(r^{\prime}\right) \cdot q_{r^{\prime}}
$$

- three point distribution in $\{0, r, b\}$ :

$$
f(0) \cdot q_{0}+f(r) \cdot q_{r}+f(b) \cdot q_{b} .
$$

\section{Resulting restrictions on the tail probabilities}

\subsection{Only the mode m known}

The restrictions for this case are given in Table 1.

Table 1

\begin{tabular}{lll}
\hline Conditions & Upper bound & Lower bound \\
\hline$t \leqslant m$ & 1 & $(m-t) / m$ \\
$t>m$ & $(b-t) /(b-m)$ & 0 \\
\hline
\end{tabular}

3.2. Only the first moment $\mu_{1}$ known

The restrictions for this case are given in Table 2 .

Table 2

\begin{tabular}{lll}
\hline Conditions & Upper bound & Lower bound \\
\hline$t \leqslant \mu_{1}$ & 1 & $\left(\mu_{1}-t\right) /(b-t)$ \\
$t>\mu_{1}$ & $\mu_{1} / t$ & 0 \\
\hline
\end{tabular}


Table 3

\begin{tabular}{lll}
\hline Conditions & Upper bound & Lower bound \\
\hline $0 \leqslant t \leqslant b^{\prime}$ & 1 & $\frac{\left(\mu_{1}-t\right)^{2}}{\left(\mu_{1}-t\right)^{2}+\mu_{2}-\mu_{1}^{2}}$ \\
$b^{\prime}<t \leqslant 0^{\prime}$ & $\frac{(b+t) \mu_{1}-\mu_{2}}{b t}$ & $\frac{\mu_{2}-\mu_{1} t}{b(b-t)}$ \\
$0^{\prime}<t \leqslant b$ & $\frac{\mu_{2}-\mu_{1}^{2}}{\mu_{2}-\mu_{1}^{2}+\left(\mu_{1}-t\right)^{2}}$ & 0 \\
\hline
\end{tabular}

3.3. The two moments $\mu_{1}$ and $\mu_{2}$ known

The restrictions for this case are given in Table 3, where

$$
b^{\prime}=\left(\mu_{2}-\mu_{1} b\right) /\left(\mu_{1}-b\right) \quad \text { and } \quad 0^{\prime}=\mu_{2} / \mu_{1} \text {. }
$$

\subsection{Mode $m$ and mean $\mu_{1}$ known}

(a) $t \leqslant m$. Define

$$
c_{1} \equiv t-\sqrt{(m-t)(b-t)}
$$

Table 4 gives the relevant restrictions.

Table 4

\begin{tabular}{llll}
\hline Conditions & Upper bound & Conditions & Lower bound \\
\hline$t<v_{1}$ & 1 & $c_{1} \leqslant 0$ & $\frac{b(m-t)+v_{1} t}{b m}$ \\
$v_{1} \leqslant t$ & $\frac{m+v_{1}-t}{m}$ & $0<c_{1} \leqslant v_{1}$ & $\frac{\left(t-c_{1}\right) v_{1}+\left(b-c_{1}\right) m-b t+c_{1}^{2}}{\left(b-c_{1}\right)\left(m-c_{1}\right)}$ \\
& & $v_{1}<c_{1} \leqslant t$ & $\frac{m-t}{m-v_{1}}$ \\
\hline
\end{tabular}

(b) $t>m$. Define

$$
c_{2} \equiv t+\sqrt{t(t-m)} .
$$

Table 5 gives the relevant restrictions. 
Table 5

\begin{tabular}{llll}
\hline Conditions & Upper bound & Conditions & Lower bound \\
\hline$t \leqslant c_{2} \leqslant v_{1}$ & $\frac{v_{1}-t}{v_{1}-m}$ & $t<v_{1}$ & $\frac{v_{1}-t}{b-m}$ \\
$v_{1}<c_{2} \leqslant b$ & $\frac{v_{1}\left(c_{2}-t\right)}{c_{2}\left(c_{2}-m\right)}$ & $v_{1} \leqslant t$ & 0 \\
$b \leqslant c_{2}$ & $\frac{v_{1}(b-t)}{b(b-m)}$ & & \\
\hline
\end{tabular}

Table 6

\begin{tabular}{ll}
\hline Conditions & Upper bound \\
\hline $0 \leqslant t \leqslant b^{\prime}$ & 1 \\
$b^{\prime}<t \leqslant 0^{\prime}$ & $\frac{b(m-t)+(b+t) v_{1}-v_{2}}{b m}$ \\
$0^{\prime}<t \leqslant b$ & $\frac{1}{t-t^{\prime}}\left(\left(v_{1}-t^{\prime}\right)+\frac{\left(t-v_{1}\right)(m-t)}{m-t^{\prime}}\right)$ \\
\hline
\end{tabular}

3.5. Mode $m$ and moments $\mu_{1}$ and $\mu_{2}$ known

(a) $t \leqslant m$. Upper bounds for this case are given in Table 6 , where

$$
b^{\prime}=\left(v_{2}-v_{1} b\right) /\left(v_{1}-b\right), 0^{\prime}=v_{2} / v_{1} \quad \text { and } \quad t^{\prime}=\left(v_{2}-v_{1} t\right) /\left(v_{1}-t\right) .
$$

Lower bounds in case $\mu_{1}>m$ are to be treated now. Consider $b^{\prime}=\left(v_{2}-v_{1} b\right) /\left(v_{1}-b\right)$ and $0^{\prime}=v_{2} / v_{1}$. Define

$$
s \equiv \frac{b t+m t-b m-\sqrt{m b(b-t)(m-t)}}{t},
$$

and calculate $r$ as the unique root in $\left[0, \min \left(b^{\prime}, t\right)\right]$ of $r^{3}+A r^{2}+B r+C=0$ with

$$
\begin{aligned}
& A=-\frac{1}{2}\left(2 v_{1}+m+3 t\right), \\
& B=2 t v_{1}+t m, \\
& C=\frac{1}{2}\left(v_{2} m-v_{2} t-2 v_{1} t m\right) .
\end{aligned}
$$

The lower bounds obtained are given in Table 7 . 
Table 7

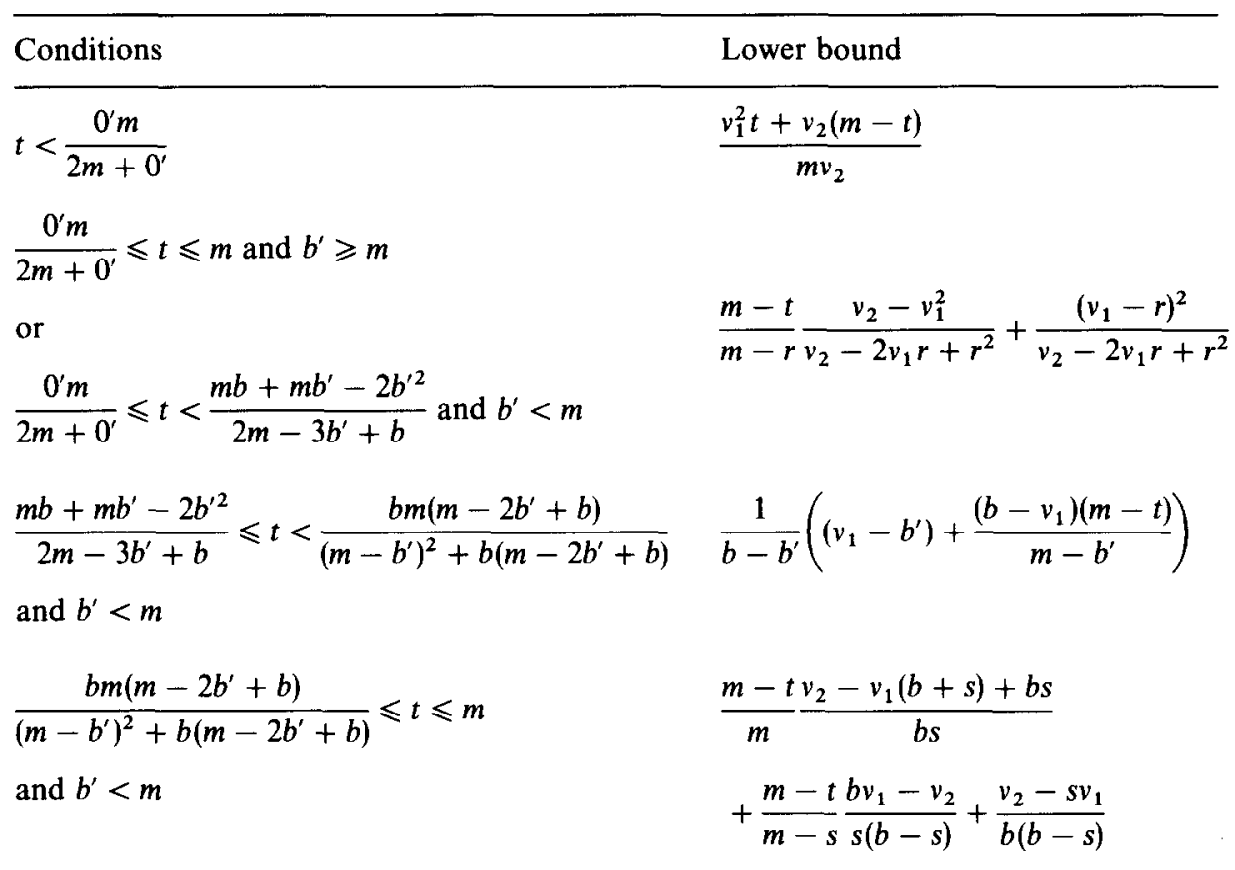

Table 8

\begin{tabular}{ll}
\hline Conditions & Lower bound \\
\hline $0 \leqslant t \leqslant b^{\prime}$ & $\frac{\left(v_{1}-t\right)^{2}}{\left(v_{1}-m\right)\left(v_{1}-t\right)+v_{2}-v_{1}^{2}}$ \\
$b^{\prime}<t \leqslant 0^{\prime}$ & $\frac{v_{2}-v_{1} t}{b(b-m)}$ \\
$0^{\prime}<t \leqslant b$ & 0 \\
\hline
\end{tabular}

Lower bounds in case $\mu_{1} \leqslant m$ are to be dealt with now. Define

$$
m^{G} \equiv b-m, \quad t^{G} \equiv b-t, \quad \mu_{1}^{G} \equiv b-\mu_{1} \quad \text { and } \quad \mu_{2}^{G} \equiv b^{2}-2 b \mu_{1}+\mu_{2}
$$

Determine the upper bound for this new distribution (for which $\mu_{1}^{G}>m^{G}$ ). The lower bound for the old distribution can then be found by subtracting this result from 1 . 
(b) $t>m$. Lower bounds for this case are provided in Table 8, where

$$
b^{\prime}=\left(v_{2}-v_{1} b\right) /\left(v_{1}-b\right) \text { and } 0^{\prime}=v_{2} / v_{1} \text {. }
$$

Upper bounds in case $\mu_{1}>m$ are to be derived now. Consider $b^{\prime}=\left(v_{2}-v_{1} b\right) /\left(v_{1}-b\right)$ and $0^{\prime}=v_{2} / v_{1}$. Define

$$
s \equiv \frac{t(b-m)+\sqrt{b t(b-m)(t-m)}}{b-t} \quad \text { and } \quad c_{3} \equiv t+\sqrt{t(t-m)}
$$

and calculate $r$ as the unique root in $\left[\max \left(0^{\prime}, t\right), b\right]$ of $r^{3}+A r^{2}+B r+C=0$ with

$$
\begin{aligned}
& A=-\frac{1}{2}\left(2 v_{1}+m+3 t\right), \\
& B=2 t v_{1}+t m, \\
& C=\frac{1}{2}\left(v_{2} m-v_{2} t-2 v_{1} t m\right) .
\end{aligned}
$$

Now upper bounds given in Table 9 can be obtained.

Table 9

$$
\begin{array}{ll}
\hline \text { Conditions } & \text { Upper bound } \\
\hline m<t \leqslant \frac{b b^{\prime 2}}{\left(b^{\prime}-m\right)^{2}+b\left(2 b^{\prime}-m\right)} \text { and } b^{\prime}>c_{3} & \frac{1}{b-b^{\prime}}\left(\frac{\left(v_{1}-b^{\prime}\right)(b-t)}{b-m}+\frac{\left(b-v_{1}\right)\left(b^{\prime}-t\right)}{b^{\prime}-m}\right) \\
m<t \leqslant \frac{b 0^{\prime 2}}{\left(0^{\prime}-m\right)^{2}+b\left(20^{\prime}-m\right)} \text { and } b^{\prime} \leqslant c_{3} & \frac{1}{b-s}\left(\frac{\left.b v_{1}-v_{2}\right)(s-t)}{s(s-m)}+\frac{\left(v_{2}-v_{1} s\right)(b-t)}{b(b-m)}\right) \\
\text { or } \quad & \\
\frac{b b^{\prime 2}}{\left(b^{\prime}-m\right)^{2}+b\left(2 b^{\prime}-m\right)}<t \leqslant \frac{b 0^{\prime 2}}{\left(0^{\prime}-m\right)^{2}+b\left(20^{\prime}-m\right)} & \\
\text { and } b^{\prime}>c_{3} & \frac{v_{1}^{2}\left(v_{2}-t v_{1}\right)}{v_{2}\left(v_{2}-m v_{1}\right)} \\
\frac{b 0^{\prime 2}}{\left(0^{\prime}-m\right)^{2}+b\left(20^{\prime}-m\right)}<t \leqslant \frac{20^{\prime 2}-m 0^{\prime}}{30^{\prime}-2 m} & \frac{r-t}{r-m} \frac{v_{2} \quad v_{1}^{2}}{r^{2}-2 v_{1} r+v_{2}} \\
\frac{20^{\prime 2}-m 0^{\prime}}{30^{\prime}-2 m}<t \leqslant \frac{2 b^{2}-b m-b^{\prime} m}{3 b-b^{\prime}-2 m} & \frac{b-t}{b-m} \frac{v_{2}-v_{1}^{2}}{b^{2}-2 v_{1} b+v_{2}} \\
\frac{2 b^{2}-b m-b^{\prime} m}{3 b-b^{\prime}-2 m}<t \leqslant b &
\end{array}
$$




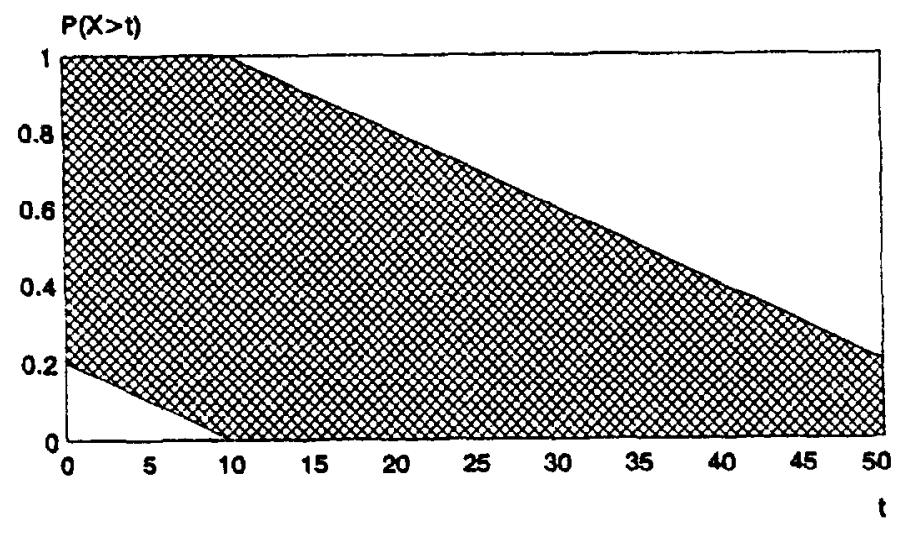

Range $[0,50] \mu 1=10$

Fig. 1. Restrictions on tail probabilities in case moment $\mu_{1}$ is known.

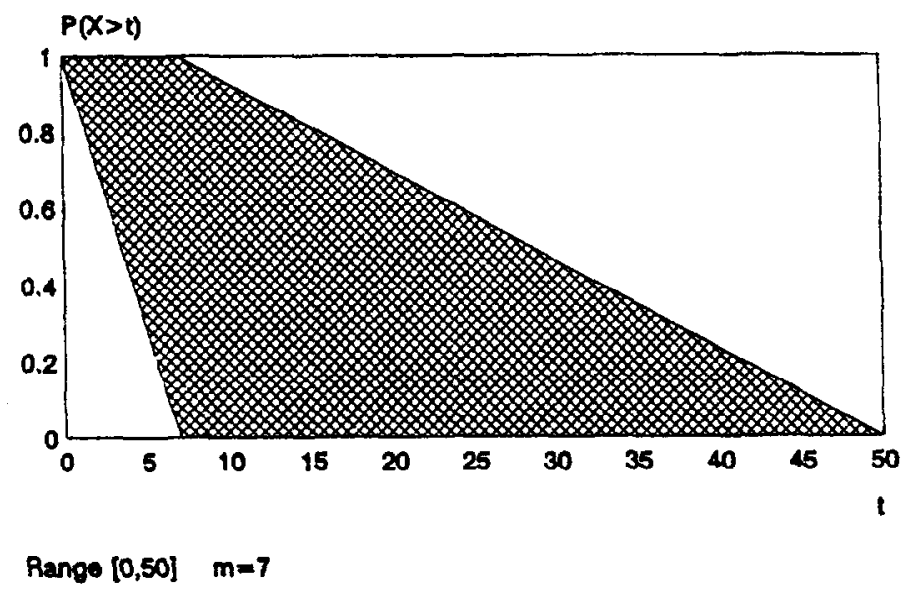

Fig. 2. Restrictions on tail probabilities in case mode $m$ is known.

Upper bounds in casc $\mu_{1} \leqslant m$ are to be dealt with. For this definc

$$
m^{G} \equiv b-m, \quad t^{G} \equiv b-t, \quad \mu_{1}^{G} \equiv b-\mu_{1} \quad \text { and } \quad \mu_{2}^{G} \equiv b^{2}-2 b \mu_{1}+\mu_{2}
$$

Determine the lower bound for this new distribution (for which $\mu_{1}^{G}>m^{G}$ ). The upper bound for the old distribution can then be found by subtracting this result from 1 . 


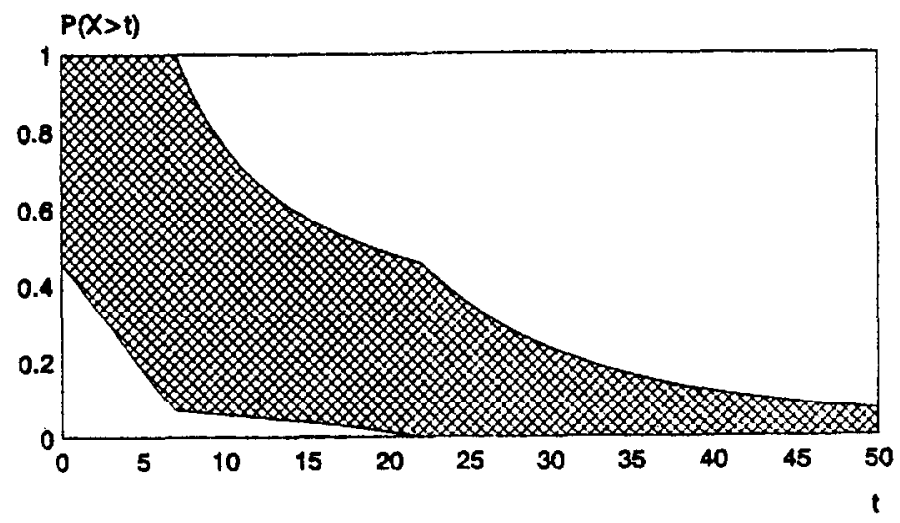

Range $[0,50] \mu 1=10 \quad \mu 2=220$

Fig. 3. Restrictions on tail probabilities in case moments $\mu_{1}$ and $\mu_{2}$ are known.

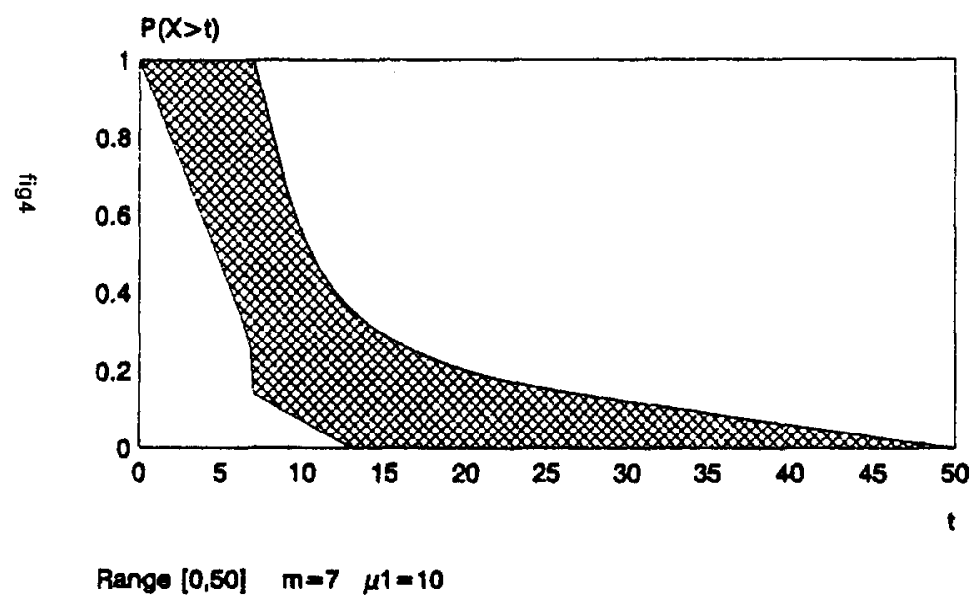

Fig. 4. Restrictions on tail probabilities in case mode $m$ and moment $\mu_{1}$ are known.

\section{Numerical examples}

In this section, we calculate the extreme values for tail probabilities for two different portfolios; one is meant to show the different possibilities, while the other concerns the problem already mentioned in the Introduction.

The first portfolio has range $b=50$, mode $m=7$, and moments $\mu_{1}=10$ and $\mu_{2}=220$. Figs. $1-5$ show the range that contains all values that can be obtained by the tail probability $P(X \geqslant t)$ as 


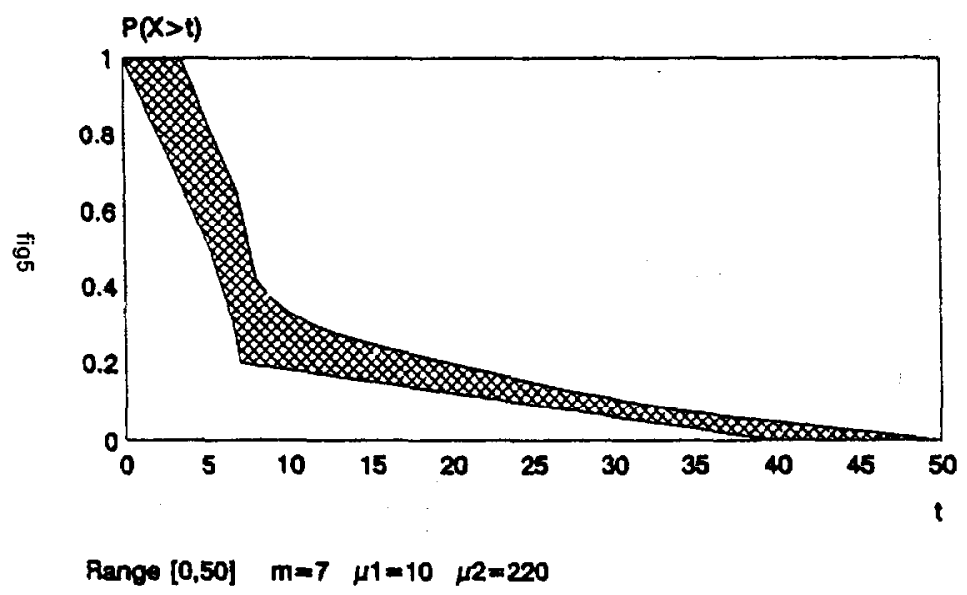

Fig. 5. Restrictions on tail probabilities in case mode $m$, moments $\mu_{1}$ and $\mu_{2}$ are known.

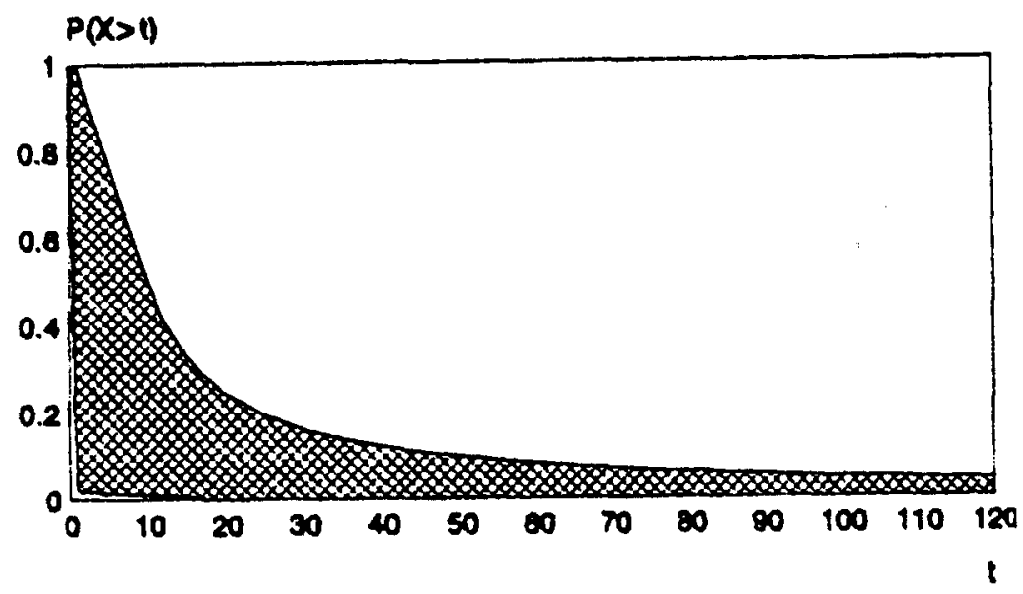

Range $[0,1000] \quad m=1 \quad \mu t=10$

Fig. 6. Restrictions on tail probabilities (first part).

a function of $t$, for the different cases of knowledge of the characteristics for the unknown distribution.

It is interesting to remark that the region in Fig. 5 cannot be found as a simple intersection of the regions in Figs. 2 and 3, because the various conditions are not mutually independent.

For the second example, we look at the portfolio considered in [4]. Here $b=1000, m=1$ and $\mu_{1}=10$. Figs. 6 and 7 deal with the situation of knowledge of all these three parameters. 


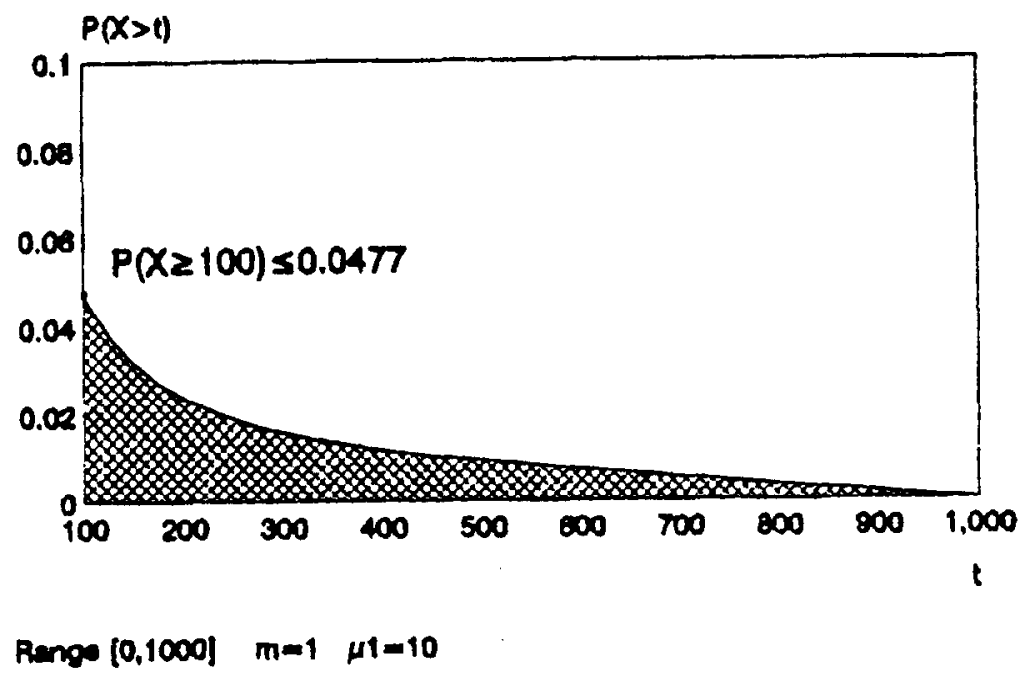

Fig. 7. Restrictions on tail probabilities (second part).

\section{References}

[1] P.L. Brockett and S.H. Cox Jr, Insurance calculations using incomplete information, Scand. Actuar. J. 15 (1985) 94-108.

[2] A. De Schepper and B. Heijnen, Extreme values of tail probabilities, Working Paper 91/11 RUCA, Antwerp, 1991.

[3] W. Feller, Introduction to Probability Theory and its Applications, Vol. 2 (Wiley, New York, 1971).

[4] M.J. Goovaerts, J. Haezendonck and F. De Vylder, Numerical best bounds on stop-loss premiums, Insurance Math. Econom. 1 (1982) 287-302.

[5] B. Heijnen, Best upper and lower bounds on modified stop-loss premiums in case of known range, mode, mean and variance of the original risk, Insurance Math. Econom. 9 (1990) 207-220. 